\title{
Neuromyelitis optica - A masquerade of multiple sclerosis
}

\author{
Kambam Gainathi ${ }^{1, *}$, Elfride Farokh Sanjana ${ }^{2}$, Hannah Ranjee Prasanth ${ }^{3}$ \\ ${ }^{1}$ Post Graduate, ${ }^{2}$ Professor \& HOD,${ }^{3}$ Associate Professor, Dept. of Ophthalmology, Pondicherry Institute of Medical Sciences, \\ Pondicherry, India
}

*Corresponding Author:

Email: gainathireddy@gmail.com

\begin{abstract}
We describe a case of neuromyelitis optica - a Masquerade of multiple sclerosis. Neuromyelitis optica should be differentiated from multiple sclerosis, as treatment of multiple sclerosis can worsen symptoms of neuromyelitis optica. Both visual and neurological prognosis in neuromyelitis optica are poorer than in multiple sclerosis. Episodes of visual loss in neuromyelitis optica are recurrent leading to severe visual impairment. Immunosuppressive agents such as corticosteroids remain the mainstay of therapy for acute episodes.
\end{abstract}

Keywords: Aquaporin-4, Azathioprine, Multiple sclerosis, MRI, Neuromyelitis optica.

\section{Introduction}

Neuromyelitis optica is a rare inflammatory autoimmune disorder of central nervous system that commonly presents with optic neuritis or transverse myelitis. ${ }^{1}$ Earlier neuromyelitis optica was considered as a variant of multiple sclerosis, but now it is clearly recognized as a separate disorder with distinct clinical, radiographical, pathological and serological features. ${ }^{2}$ Therefore early diagnosis and aggressive treatment is critical for the preservation of visual and neurological function. ${ }^{3}$

\section{Materials and Methods}

An 18 year old female presented with history of fever since one week, weakness of lower limbs since 5 days, sudden, profound, painless diminished vision in LE since 2 days. She was diagnosed to have Acute Demyelinating Encephalomyelitis (ADEM) and was on treatment with steroid, T. Prednisolone $60 \mathrm{mg}$ OD initially with tapering dose and immunosuppressant $\mathrm{T}$. Azathioprine 50mg BD. On presentation BCVA OD was $6 / 6$ with rest of the anterior segment being unremarkable. BCVA OS was PL present, PR inaccurate in nasal quadrant. Stereoscopic dilated fundus examination was normal in the right eye. Temporal pallor of the disc present in the left eye (Fig. 1) with rest of the fundus being normal. MRI brain showed mild focal cerebral edema in bilateral frontal regions with effacement of sulcal spaces and hyperintense lesions in right occipital lobe (Fig. 2). MRI spinal cord showed multiple short segment intrameduallary plaques between $\mathrm{C} 2-\mathrm{C} 7$ segments (Fig. $3)$. She gave prior history of recurrent episodes of fever, headache, difficulty in voiding urine, weakness \& diminished sensation over lower limbs with episodes of seizure. MRI done at that time, one year ago showed hyperintense lesions in right peritrigonal, body of corpus callosum on right side and left middle cerebellar peduncle, multiple short segment intramedullary plaques in cervical spinal cord with no cord expansion.
Antibodies to aquaporin-4 was done which was negative. For the present episode, she was started on immunoglobulins and physiotherapy with symptomatic improvement. Treatment with Inj. methyl prednisolone (1gram IV OD /day for 3 days ) followed by oral tablet prednisolone $60 \mathrm{mg}$ was initiated along with anticonvulsant, T. Levetiracetam 500mg BD and immunosuppressant T.Azathioprine 50mg BD. Vision improved to $6 / 18$ at the end of treatment.

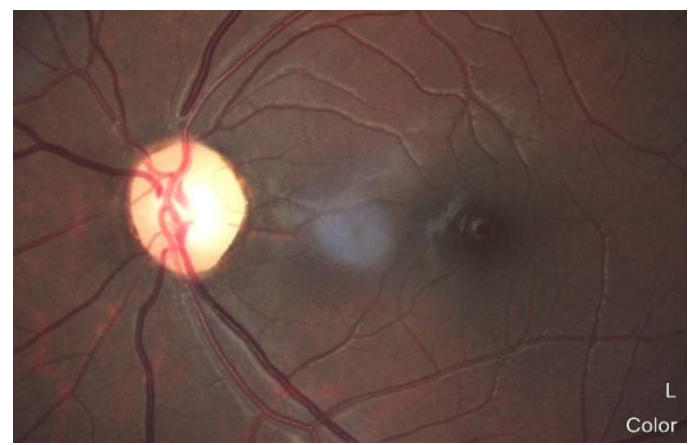

Fig. 1: Showing temporal pallor of the disc in left eye

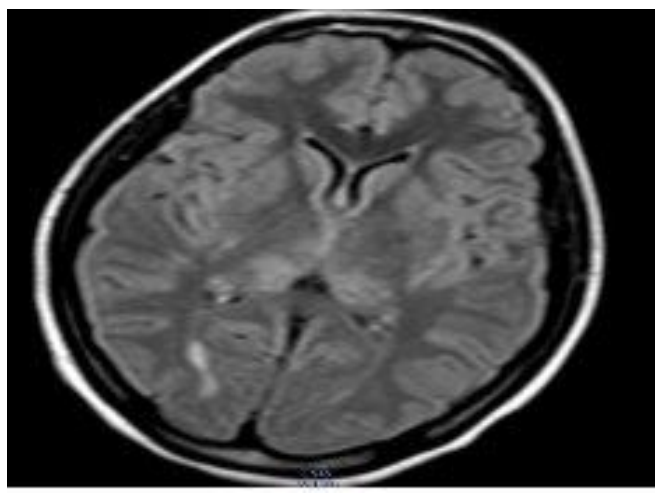

Fig. 2: Showing mild focal cerebral edema in bilateral frontal regions with effacement of sulcal spaces. Hyperintense lesion in right occipital lobe with normal ventricles 


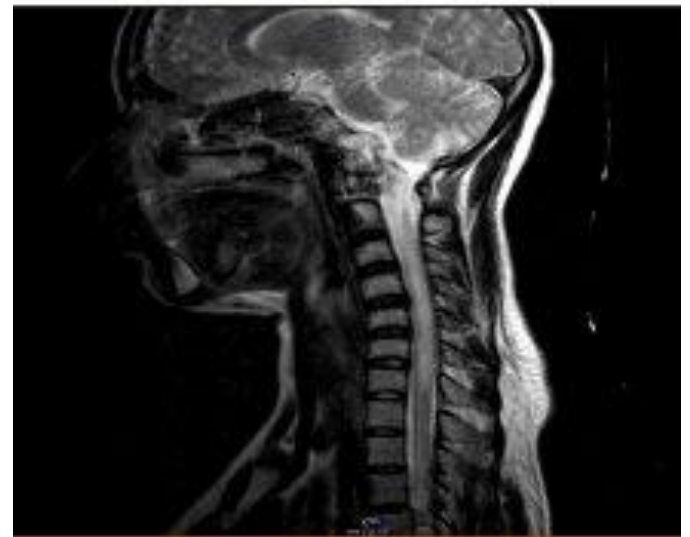

Fig. 3: Showing multiple short segment intrameduallry plaques in cervical spinal cord between $\mathrm{C} 2-\mathrm{C} 7$ segments

\section{Discussion}

NMO-IgG antibodies are produced against aquaporin-4 which is a CNS water channel present in the astrocyte foot processes at the blood brain barrier helping to maintain water hemostasis. ${ }^{4}$ The high specificity of AQP4-IgG for NMO has allowed the identification of seropositive patients with atypical presentations of the disease called NMO spectrum disorders (NMOSD). ${ }^{1}$

Brain MRI lesions in multiple sclerosis are confined to periventricular or subcortical regions whereas in neuromyelitis optica are symmetrical hypothalamic or brainstem lesions. Spinal cord MRI lesions are seen in 1-2 segments in multiple sclerosis and in neuromyelitis optica more than three segments are involved. During relapses of multiple sclerosis CSF WBC is $<50 / \mathrm{mm}^{3}$, in neuromyelitis optica it is often $>50 / \mathrm{mm}^{3}$. CSF oligoclonal bands in multiple sclerosis are about $85 \%$ and in neuromyelitis optica are about $15-30 \%$. Multiple sclerosis is not usually associated with auto immune disorders and neuromyelitis optica is commonly associated with systemic immune disease. Severity of relapses in multiple sclerosis is usually mild to moderate with fair to good recovery but in neuromyelitis optica severity of relapses is usually moderate to severe with poor recovery. Interferon therapy is helpful in treating multiple sclerosis whereas interferon therapy worsens neuromyelitis optica. ${ }^{2}$

Revised Diagnostic criteria for neuromyelitis optica includes: ${ }^{5}$

Required (at least 1 attack of each)

1. Optic neuritis.

2. Transverse myelitis.

Supportive (2 out 3 )

1. Brain MRI - normal or lesions not meeting MS criteria.

2. Spinal cord MRI - lesions extending continuously over 3 or more segments.
3. NMO IgG seropositivity.

Acute attacks are treated with IV Methylprednisolone 1000mg daily for 3-5 days. Intravenous immunoglobulin and cyclophosphamide have also been used to treat acute exacerbations and prevent relapses. Recurrent attacks can be prevented with corticosteroids with a maintance dose of more than $10 \mathrm{mg} / \mathrm{day}^{2}{ }^{2}$ Azathioprine and mycophenolate mofetil are the most commonly used first-line therapies. ${ }^{3}$ However, some patients experience a relapse following treatment. Patients with a pre-treatment history of a severe attack and a younger age of onset exhibited an increased risk of a poor response to Azathioprine/mycophenolate therapy. ${ }^{3}$ Methotrexate, mitoxantrone, rituximab, eculizumab, are also used. Aggressive treatment of relapses and prophylactic immunomodulatory therapy are the focus of treatment. Immunosuppressive agents such as corticosteroids remain the mainstay of therapy for acute episodes. Long term immunosuppressive therapy with azathioprine, mycophenolate and rituximab should be considered, particularly if the patient is NMO-IgG positive. The duration of treatment is usually not less than 24 months. ${ }^{6}$

In a study done by Eskandarieh $\mathrm{S}$, Nedjat $\mathrm{S}$ et al in 2016 to study the prevalence of NMO-SD in Tehran, the prevalence was 0.86 per 100,000 with female to male ratio of 5:1. NMO-IgG was positive in $46.8 \%$ patients, with the primary presenting symptom being transverse myelitis which was observed in $28.2 \%$ patients. $^{7}$

In a study done to find aquaporin 4 antibody in Severe Optic Neuritis Patients in India, NMO antibody testing was performed in 40 patients with the atypical severe optic neuritis in patients with age between 10 and 68, NMO antibody was positive in 8 of the 40 patients (20\%). All the 8 patients who were seropositive were females with statistically significant $p$ value of $0.037 .^{8}$

\section{Conclusion}

This case is being presented to highlight the existence of a neurological entity-NMO spectrum disorders (NMOSD). Both neurological and visual prognosis in NMO are poorer than multiple sclerosis. NMO should be differentiated from multiple sclerosis as treatment for multiple sclerosis can make NMO symptoms worse.

\section{References}

1. Han J, Yang M, Zhu J, Jin T. Complexity and wide range of neuromyelitis optica spectrum disorders: more than typical manifestations. Neuropsychiatr Dis Treat. 2017 Oct 20;13:2653-60.

2. Kowarik MC, Soltys J, Bennett JL. The Treatment of Neuromyelitis Optica. J Neuroophthalmol. 2014 Mar;34(1):70-82.

3. Kim S-H, Hyun J-W, Joung A, Park EY, Joo J, Kim HJ. Predictors of response to first-line immunosuppressive 
therapy in neuromyelitis optica spectrum disorders. Mult Scler J. 2017 Jan 12;23(14):1902-8.

4. Roy U, Saini DS, Pan K, Pandit A, Ganguly G, Panwar A. Neuromyelitis Optica Spectrum Disorder with Tumefactive Demyelination mimicking Multiple Sclerosis: A Rare Case. Front Neurol. 2016 May 11;7:73.

5. Wingerchuk DM, Lennon VA, Pittock SJ, Lucchinetti $\mathrm{CF}$, Weinshenker BG. Revised diagnostic criteria for neuromyelitis optica. Neurology. 2006 May 23;66(10):1485-9.

6. Miller NR, Walsh FB, Hoyt WF. Walsh and Hoyt's clinical neuro-ophthalmology. Vol. 2. Lippincott Williams \& Wilkins; 2005.

7. Eskandarieh S, Nedjat S, Azimi AR, Moghadasi AN, Sahraian MA. Neuromyelitis optica spectrum disorders in Iran. Mult Scler Relat Disord. 2017 Nov 29;18:209-12.

8. Ambika S, Balasubramanian M, Theresa L, Veeraputhiran A, Arjundas D. Aquaporin 4 antibody [NMO Ab] status in patients with severe optic neuritis in India. International ophthalmology. $2015 \mathrm{Dec}$ 1;35(6):801-6. 